\title{
NeURocisticercose gigante: Diagnóstico e tratamento
}

\author{
Rafael Augusto Castro Santiago Brandão ${ }^{*}$, Tadeu Wilker Nunes ${ }^{2}$, Marcos Antonio Dellaretti Filho ${ }^{3}$, Paolo Victor Fernandes Tótola ${ }^{2}$, Vinícius Silveira Fonseca², \\ Warley Cristiano de Souza ${ }^{2}$ \\ Trabalho realizado no Hospital Santa Casa de Belo Horizonte, Belo Horizonte, MG
}

A neurocisticercose (NCC) é a mais comum infecção parasitária do sistema nervoso ${ }^{1}$. Considerada uma epidemia mundial, a parasitose apresenta-se, sobretudo, na forma assintomática ${ }^{2}$. Em geral o tratamento é clínico, mas em alguns casos selecionados a intervenção cirúrgica é uma boa opção de tratamento. No caso relatado, tendo-se em vista a magnitude da lesão intracraniana, a intervenção cirúrgica foi a conduta terapêutica escolhida.

Paciente do sexo feminino, 54 anos, feoderma, natural de Novo Cruzeiro (MG) e procedente de Belo Horizonte. Apresentou quadro de cefaleia frontal de forte intensidade, evoluindo com hemiparesia direita e crise convulsiva. Dez dias após o início dos sintomas, procurou o serviço de pronto-atendimento. A tomografia de crânio (TC) revelou área de hipodensidade em região frontoparietal esquerda com características de lesão cística(Figuras 1A e 1B).

A intervenção cirúrgica foi escolhida para o caso. Foi realizada uma pequena craniotomia frontal com aspiração de líquido claro intracístico e exérese da cápsula com o escólex Cysticercus cellulosae (Figura 2A e 2B). Após o ato cirúrgico, o tratamento farmacológico utilizado foi albendazol $15 \mathrm{mg} / \mathrm{kg} /$ dia durante 28 dias em combinação com dexametasona $4 \mathrm{mg}$ $6 / 6$ horas.

Após um ano de follow up, a paciente apresentou melhora do déficit motor, mas mantém queixa de vertigens e cefaleia. TC de controle evidenciou área de encefalomalácia residual, sem sinais de novos cisticercos. (CC) (Figuras 3A e 3B).

A forma de NCC constituída por um único cisto gigante é considerada rara. Em um levantamento epidemiológico de janeiro 1980 a dezembro de 2001 realizado pelo Hospital das Clínicas da Faculdade de Medicina de Ribeirão Preto (HC-FMRP), foi identificado incidência de $12,9 \%$ de cistos gigantes - forma tumoral - entre os pacientes acometidos por NCC cerebral ${ }^{3}$. Cistos gigantes são lesões com mais de 5 centímetros em seu maior diâmetro e são da forma racemosa ${ }^{3,4}$. A TC apresenta característica compatível com lesão cisticercoide, visto que seu coeficiente de atenuação é semelhante ao do líquido cefalorraquidiano (LCR), com cistos uni ou multiloculados, com pouca ou nenhuma captação de contraste ao redor. ${ }^{1}$ O tratamento pode ser cirúrgico ou clínico. O tratamento de primeira escolha na NCC é o medicamentoso e inclui drogas antiparasitárias e medicação sintomática. Praziquantel e albendazol são drogas com ação antiparasitárias contra o CC da
Taenia solium comumente utilizadas, com eficácia de 60 a $85 \%$, principalmente sobre cistos parenquimatosos, sendo que o albendazol é o tratamento de escolha pela sua maior eficácia e menor efeito colateral ${ }^{3}$. Estudos recentes apontam que a escolha do medicamento antiparasitário deve ser influenciada mais pelos custos e efeitos colaterais, uma vez que diversas análises mostram eficácia de tratamento semelhante entre 0 albendazol e o praziquantel ${ }^{5}$.

Além de matar o CC, o objetivo do tratamento é melhorar a sintomatologia clínica do paciente. Del Brutto reafirma, por meio da avaliação de estudos pregressos, a validade do tratamento antiparasitário na melhor resolução das lesões císticas, bem como na diminuição dos episódios sintomáticos. Em um estudo realizado por Garcia ${ }^{7}$ foi evidenciado que o uso de albendazol reduziu em $46 \%$ a taxa de convulsões quando comparado ao placebo. Essas conclusões são baseadas no estudo de pacientes com cistos parenquimatosos viáveis, que representam a maioria dos pacientes com NCC na América Latina ${ }^{7}$. Esse tipo de tratamento medicamentoso reduz a duração dos fenômenos neuroimunológicos envolvidos na doença, mas devido à morte do CC e a indução de inflamação local com consequente aumento da sintomatologia neurológica, o uso de corticoides geralmente é indicado para controlar fenômenos como edema e hipertensão intracraniana (HIC). Apesar de amplamente utilizada, estratégia terapêutica com antiparasitários tem sido questionada e extensamente discutida. Nesse âmbito, torna-se evidente a necessidade de novas abordagens terapêuticas, mais seguras e de melhor eficácia 8,9 .

Apesar da eficácia do praziquantel e do albendazol, a remoção cirúrgica tem sido um tratamento utilizado para casos de cistos gigantes, principalmente naqueles em que necessitam de um diagnóstico diferencial ${ }^{4}$. Cistos localizados nos ventrículos cerebrais ou cisternas basais cerebrais não respondem bem ao tratamento clínico, sendo necessária remoção cirúrgica na maioria dos casos, podendo ser realizada por endoscopia $^{10}$. Na NCC intraparenquimatosa, o tratamento cirúrgico está indicado em casos de cistos gigantes que evoluem com sinais clínicos resultantes de compressão local do encéfalo e dos nervos cranianos, as formas que evoluem com $\mathrm{HIC}^{3} \mathrm{e}$ em casos que não há a diminuição da pressão intracraniana mesmo com o uso de corticoesteroides ${ }^{4}$. É indicado também, tal procedimento, em situações nas quais há aumento radiográfico da lesão, ataques epilépticos descontrolados ou déficit

\footnotetext{
1. Residente em Neurocirurgia na Santa Casa de Belo Horizonte, Belo Horizonte, MG

2. Acadêmicos do nono período de Medicina da Faculdade de Medicina da Universidade Federal de Minas Gerais - UFMG, Belo Horizonte, MG

3. Neurocirurgião do Hospital Santos Casa de Belo Horizonte, Belo Horizonte, MG
} 


\section{Figura 1 - A - TC sem contraste, evidenciando lesão cística em região frontoparietal.B - TC com contraste, evidenciando lesão cística sem captação}

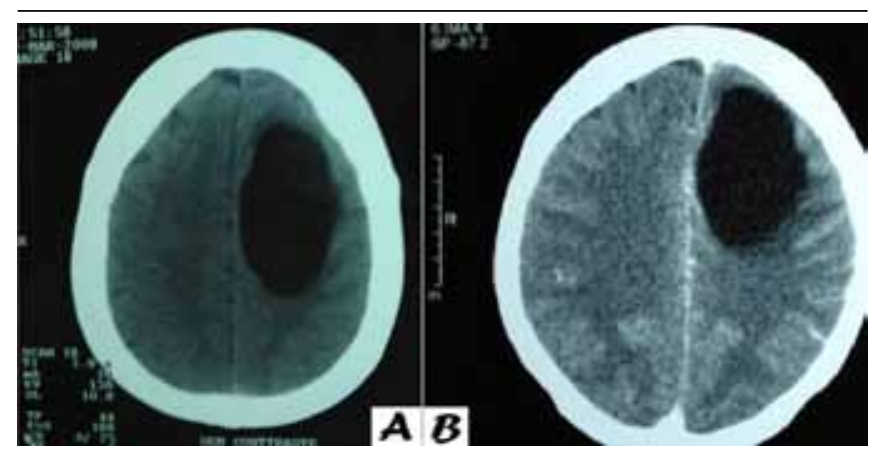

Figura 2 - A - Escólex e cápsula retirados no ato operatório, comprovando, definitivamente, que se trata de um caso de neurocisticercose B - Microscopia de cisto (Cysticercus cellulosae) com escólex invaginado de vesícula cisticercoide

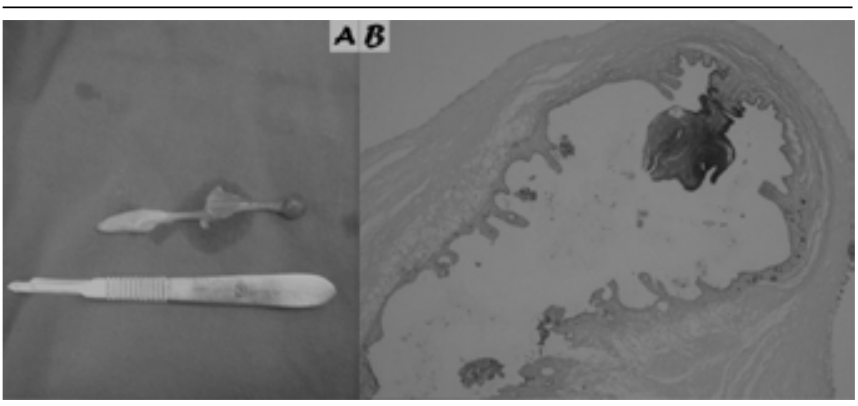

Figura 3 - A- TC de crânio com área de encefalomalácia residual B - Ausência de novos cisticercos

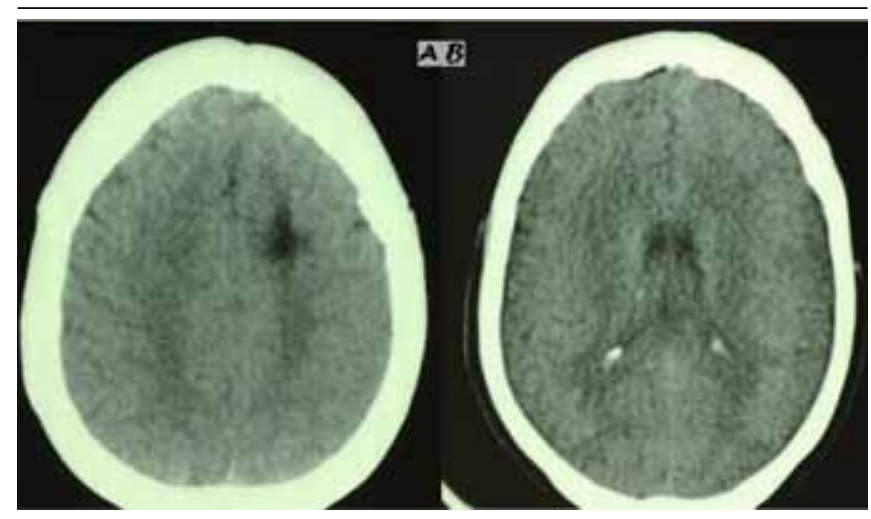

neurológico focal progressivo ${ }^{11}$. Colli ainda cita que o melhor tratamento para a forma tumoral da NCC é a exérese cirúrgica por acesso direto ${ }^{12}$. No caso relatado optou-se por tratamento cirúrgico da lesão ${ }^{13}$. Essa cirurgia visa a exérese dos cistos por abordagem direta, ou pelo menos à sua ressecção parcial, e é realizada com relativa facilidade quando o cisto gigante está localizado no parênquima e está na sua fase ativa, pois ainda está frouxamente aderido.

O tratamento medicamentoso também é uma opção para cistos gigantes em pacientes estáveis do ponto de vista neurológico. Proaño ${ }^{4}$ tratou 33 pacientes com cistos cerebrais maiores de 5 centímetros com albendazol ( $15 \mathrm{mg} / \mathrm{kg}$ por um mês) ou praziquantel (100mg por kg por um mês) sendo que 17 pacientes receberam dois ciclos de tratamento, três receberam três ciclos e um recebeu quatro ciclos. Em todos os pacientes ocorreu a calcificação ou desaparecimento do cisto em um follow up de 59 meses. Dos 22 pacientes com convulsões, 11 apresentaram remissão das crises convulsivas.

Embora no caso em estudo o tratamento de escolha foi o cirúrgico, ainda não há evidências científicas suficientes para preferir esse tratamento ou o medicamentoso. No caso relatado, tendo-se em vista a magnitude da lesão intracraniana e a sintomatologia de compressão do parênquima cerebral pelo cisto gigante, com instabilidade importante do quadro neurológico, a intervenção cirúrgica é a conduta terapêutica mais recomendada ${ }^{13}$.

A NCC é uma doença relativamente prevalente no nosso meio. Em geral o tratamento é clínico, mas em alguns casos selecionados a intervenção cirúrgica é uma boa opção.

\section{REFERÊNCIAS}

1. Cerdas C, Retana M, Ramírez G, Valenciano A. Neurocisticercosis parenquimatosa activa: reporte de um caso y revisión de la literatura. Rev Costarica Ciênc Med. 2004;25:41-7.

2. Medina TM, Degiorgio CM. Introduction to neurocysticercosis: aworld wide epidemic. Neurosurg Focus. 2002,12:e1.

3. Colli BO, Carlotti Jr CG. Fisiopatologia, diagnóstico e tratamento da cisticercose do sistema nervoso central. Temas Atuais Neurocirurgia. 2003;1:4-28.

4. Proaño JV, Madrazo I, Avelar F, Díaz G, López-Félix B, Díaz G, Grijalva J. Medical treatment for neurocysticercosis characterized by giant subarachnoid cysts. N Engl J Med. 2001;345:879-85.

5. Garcia HH. Antiparasitic drugs in neurocysticercosis: albendazole or praziquantel? Expert Rev Anti Infect Ther. 2008;6:295-8.

6. Del Brutto O, Ross K, Coffey C, Gárcia HH. Cysticidal drugs for neurocysticercosis: albendazole and praziquantel. Ann Intern Med. 2006;145:43-51.

7. Garcia HH, Pretell EJ, Gilman, RH, Martinez SM, Moulton LH, Del Brutto $\mathrm{OH}$, Herrera $\mathrm{G}$, et al. A trial of antiparasitic treatment to reduce the rate of seizures due to cerebral cysticercosis. N Engl J Med. 2004;350:249-58.

8. Carpio A, Kelvin EA, Bagiella E, Leslie D, Leon P, Andrews H, Hauser WA, et al. The effects of albendazole treatment on neurocysticercosis: a randomized controlled trial. J Neurol Neurosurg Psychiatry. 2008;79:1050-5.

9. Garg RK. Treatment of neurocysticercosis: is it beneficial? Expert Rev Anti Infect Ther. 2008;6:435-40.

10. Goel RK, Ahmad FU, Vellimana AK, Suri A, Chandra PS, Kumar R, et al. Endoscopic management of intraventricular neurocysticercosis. J Clin Neurosci. 2008;15:1096-101.

11. Garg RK. Single enhancing computerized tomography-detected lesion in immunocompetent patients. Neurosurg Focus. 2002;12:4.

12. Colli BO, Carlotti CG, Machado HR, Valença M, Assirati JA Jr, Amato MC. Surgical treatment of cerebral cysticercosis: long-term results and prognostic factors. Neurosurg Focus. 2002;12:e3.

13. Rocha Jr MA, Costa BS, Rocha CF, Rocha MA, Rocha CF, Carvalho GT, et al. Treatment of cerebral cysticercosis with albendazole in elevated dosages. Arq Neuropsiquiatria. 2006;66:114-6.

\section{*Correspondência:}

Rua Genebra, 936 -

Nova Suiça

Belo Horizonte - MG

CEP: 30421-121

Telefone: (31) 3334-3824 - Fax: (31) 3292-1967

rafabrand@hotmail.com 